\title{
A High-Power Microwave Reflectarray Antenna Based on Perforated Dielectric Substrate
}

\author{
Sahar Abdi Tazehabadi ${ }^{1,2}$, Shahrokh Jam ${ }^{1 *}$ \\ ${ }^{1}$ Department of Electrical Engineering, Shiraz University of Technology, Shiraz, I.R.IRAN \\ ${ }^{2}$ Mechanic Institute, Iranian Space Research Center, Shiraz, I.R.IRAN \\ *corresponding author, E-mail: jamesutech.ac.ir
}

\begin{abstract}
A high power microwave antenna based on the reflectarray concept is designed and investigated in this paper. The reflectarray aperture is directly driven by an azimuthally symmetric mode, and a directional boresight beam is realized through introducing a phase shift of $90^{\circ}$ to the phase shift profile of the reflectarray unit cells, azimuthally. A sample model operating at $\mathrm{X}$-band is designed to verify the validity of the proposed approach. Variable diameter air-filled holes through a host dielectric material are exploited as the phaseshifting unit cells due to the advantage of higher power handling capacity. Theoretical analysis and full-wave simulations are accomplished and results are in good agreement. A collimated beam of circular polarization with peak gain of $20.2 \mathrm{~dB}$ and axial ratio of 1.3 are achieved at the boresight direction for the design frequency of $10 \mathrm{GHz}$. The radiation performance of the proposed antenna as well as its compact structure makes it a potential candidate for high gain high power applications.
\end{abstract}

\section{Introduction}

The demand for high power microwave (HPM) radiating systems is growing in recent years due to their broad applications in civilian and military systems [1]. The special requirements of high power instruments place extra challenges on antenna design. In high power scenarios, the antenna is exposed to the radiation of high power sources. As a consequence, the power handling capability of the antenna becomes an important issue that must be addressed [2]. This comes along with the output mode of many of the HPM sources has azimuthal symmetry (e.g. the $\mathrm{TM}_{01}$ circular waveguide mode or the TEM coaxial mode) [3], which generates a doughnut-shaped pattern with a boresight null, if radiated directly. Several approaches for HPM antenna designs have been proposed in the literature. Mode conversion techniques $[4,5]$ are widely used to transform the undesirable azimuthally symmetric mode to one with a boresight peak (e.g. the rectangular $\mathrm{TE}_{10}$ mode or the circular $\mathrm{TE}_{11}$ mode). However, these techniques have drawbacks such as increased system losses, length, and weight. Valsov antenna [6], COBRA antenna [7], high power radial line helical array antennas $[8,9]$, and high power microwave radial line slot antenna [10] are among the antenna structures designed for certain HPM applications.
On the other hand, the reflectarray antennas are planar structures in which an array of reflective unit cells with appropriate phase responses is utilized to provide a focused or contoured beam from an illuminating wave front [11]. Reflectarray antennas have attracted great attention in recent years owing to their desirable features such as low profile, low cost, light weight, simplicity of construction, and handling [12]. This type of antennas are typically formed from locally periodic structures in which unit cells of different shapes and structures are used as spatial phase shifters [11] or spatial time-delay units [13]. The reflectarray unit cells are generally resonating or non-resonating blocks and their phase or time delay response can be controlled by modifying the geometrical parameters of element [14], changing the substrate characteristics [15], rotating the elements orientation [16], or loading the unit cell with tuning elements [17].

Many reported reflectarrays either only have limited power handling capability or are not suitable for integration with HPM systems. Most of these structures exploit metallic patterns in multi-layer configurations. Metallic grids, however, are problematic at high power, arcing occurs at field concentration points, and heating occurs due to ohmic losses experienced by strong currents in conductors [18]. Besides, in the case of direct radiation of the azimuthally symmetric output modes of HPM sources, a doughnut-shaped pattern with a boresight null will be reradiated by the reflectarray aperture, which is undesirable for high power applications.

This paper describes a dielectric reflectarray that is capable of realizing the directional radiation of azimuthally symmetric modes and has a notable power handling capability. because of the problematic effects of metallic patterns at high levels of electromagnetic energy, a dielectric approach is adopted here to design the reflectarray unit cells. A variable diameter air-filled hole through a host dielectric material $[19,20]$ is utilized as a phase-shifting unit cell to design a dielectric reflectarray antenna to operate at X-band. The directional radiation of azimuthally symmetric mode is achieved through compensating the phase differences of illuminating wave front over the reflectarray aperture. Theoretical analysis is used in conjunction with full-wave simulations to examine the radiation performance of the proposed reflectarray antenna. The results demonstrate the ability of the proposed antenna to be used for 
HPM applications.

\section{Antenna principle of operation}

Fig. 1 shows the topology of the proposed HPM dielectric reflectarray antenna. The reflectarray aperture is assumed to be located on the $\mathrm{x}-\mathrm{y}$ plane and illuminated by a conical horn antenna. In our reference scenario, the feed horn antenna is directly driven by the azimuthally symmetric $\mathrm{TM}_{01}$ circular mode and a doughnut-shaped pattern with a boresight null is radiated toward the reflectarray aperture. Since the incoming wave front has azimuthal symmetry, there is a $90^{\circ}$ phase difference between rays arriving at points of same radial distance and quadrupled azimuthal angle at the location of reflectarray aperture. To provide a boresight peak after reflection, this phase difference must be compensated by the reflectarray aperture. To achieve this, reflectarray aperture is divided into 4 equal-angle quadrants, as shown in Fig. 1. By introducing a phase shift of $90^{\circ}$ to unit cells of each quarter relative to the adjacent quadrant, directional radiation of the azimuthally symmetric wave front can be obtained by the reflectarray aperture. According to the above discussion, the phase delay from the feed horn phase center to a unit cell located at point $C(r, \varphi)$ on the reflectarray aperture is given by:

$$
\phi(r, \varphi)=-k_{0} \sqrt{F^{2}+r^{2}}-\frac{\pi(n-1)}{2}
$$

where $k_{0}$ is the propagation constant of free space, $(r, \varphi)$ are the polar coordinates of unit cell, $n$ is the number of quadrant in which the unit cell is located, and $F$ is the reflectarray focal distance. To achieve a collimated beam at the boresight direction, the phase of reflection coefficient for different unit cells over the reflectarray aperture must be selected in a way that total phase delay from feed phase center to a fixed aperture plane in front of the reflectarray becomes a constant for all unit cells. Accordingly, the required reflection phase distribution of reflectarray unit cells is determined as:

$$
\phi_{R}(r, \varphi)=k_{0} \sqrt{F^{2}+r^{2}}+\frac{\pi(n-1)}{2}+2 k \pi
$$

An X-band dielectric reflectarray antenna operating at $10 \mathrm{GHz}$ is designed, analysed, and simulated to demon-

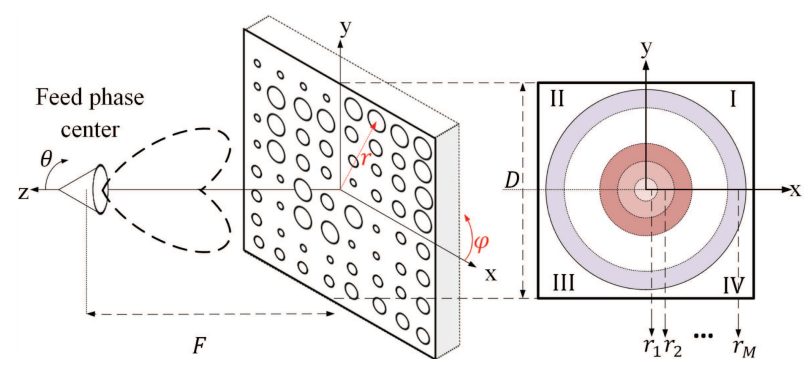

Figure 1: Topology of the proposed HPM reflectarray antenna.

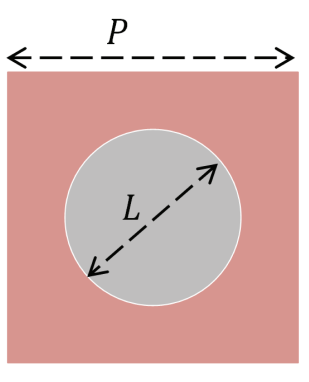

(a)
Host dielectric

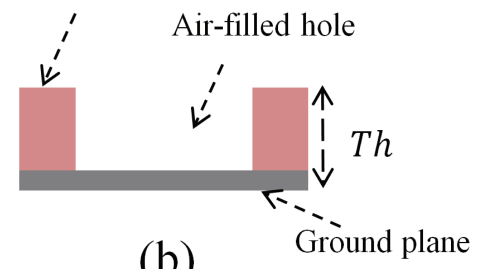

(b)
Figure 2: Structure of the dielectric unit cell. (a) Top view. (b) Side view.

strate the feasibility of the proposed approach. The dielectric unit cell is described in section 3 . In section 4, the reflectarray design procedure is presented, and the theoretical analysis and simulated results are discussed in section 5 .

\section{Reflectarray unit cell}

As mentioned previously, dielectric approach is chosen here to eliminate the problematic effects of metallic patterns in high power applications. In dielectric-type unit cell, the conductive patch is removed and the control of unit cell reflection phase is achieved by changing the parameters of the dielectric. A schematic model of the dielectric unit cell in our design is shown in Fig. 2. The unit cell is a square dielectric slab with permittivity of $\varepsilon_{r}$ that in our design covers the whole unit cell surface. The thickness of the slab is $T h$, and the unit cell periodicity is $P$.

To design the proposed HPM reflectarray, different values of the reflection phase are required for various unit cells over the reflectarray aperture. The reflection phase of unit cell can be controlled by drilling an air-filled hole that has a diameter $L$ through the dielectric slab. The diameter of the hole is then changed to provide the required reflection phase on the element position. Also, due to the reflective nature of the unit cell, a conductive ground plane has been added to the bottom side of the dielectric slab. There are three degrees of freedom to design the described unit cell. These include the permittivity and thickness of dielectric slab, and the periodicity of unit cell. In order to study the influence of these parameters on the unit cell reflection coefficient, a series of simulations are carried out utilizing the frequency domain solver of CST Microwave Studio. The unit cell is placed inside a rectangular waveguide with TEM-mode propagation boundary conditions. The top and bottom walls of the waveguide are selected as perfect electric conductors, and its side walls are selected as perfect magnetic conductors. These settings simulate a vertically polarized plane wave that incidents normally on an infinite periodic array of identical unit cells. After detailed fullwave simulations, it is found that a phase range of about $360^{\circ}$ could be achieved with a unit cell having permittivity $\varepsilon_{r}=10.2$, thickness $T h=7.62 \mathrm{~mm}$, and periodicity $P=9$ 
mm. Fig. 3 depicts the full-wave simulated reflection phase and amplitude of the unit cell versus air-filled hole diameter at $10 \mathrm{GHz}$. It can be seen that the reflection loss is less than $0.3 \mathrm{~dB}$ at the design frequency, and a phase range of about $360^{\circ}$ is achieved when the hole diameter varies from 0.1 to $8.6 \mathrm{~mm}$, which satisfies the required phase range of our dielectric reflectarray design. The phase response of unit cell at different incidence angles is also examined, and the results are shown in Fig. 4. As it can be observed, the phase response is stable for oblique incidence up to $30^{\circ}$. This proves the validity of the phase curves of Fig. 3 to be used for design the reflectarray antenna, as described in the next section.

\section{Design procedure}

The proposed approach described in section 2 was followed to design an HPM antenna to operate at a typical frequency of $10 \mathrm{GHz}$. The reflectarray has a square aperture with side length of $252 \mathrm{~mm}\left(\approx 8.2 \lambda_{0}\right)$ and thickness of $7.62 \mathrm{~mm}$ $\left(\approx 0.25 \lambda_{0}\right)$, composed of a total of $28 \times 28$ air-filled hole substrate through unit cells. Rogers 6010 dielectric substrate with relative permittivity $\varepsilon_{r}=10.2$ and loss tangent $\tan \delta=0.002$ was used for substrate layer. Circular waveguide (diameter $=27.8 \mathrm{~mm}$ ) driven from one end by $\mathrm{TM}_{01}$ mode was selected as the feed of the reflectarray. The diameter of the waveguide input aperture was selected based on the cut-off frequency of the $\mathrm{TM}_{01}$ mode. The impedance matching between the free space and waveguide output aperture for this mode was achieved through gradually expanding the guide diameter as shown in Fig. 5(a). The simulated three-dimensional far-field radiation pattern of the designed feed horn at the design frequency of 10 $\mathrm{GHz}$ is shown in Fig. 5(b). The simulated return loss of the feed horn over the frequency range of $9.5-10.5 \mathrm{GHz}$ as well as the two-dimensional plots of vertically- and horizontally polarized radiation patterns at the design frequency of $10 \mathrm{GHz}$ are shown in Fig. 5(c) and (d), respectively. The boresight nulls are clearly observed from this figure as we expected. The reflectarray focal length was chosen to be $151.2 \mathrm{~mm}$, giving a focal length to diameter ratio F/D of 0.6. The value of F/D was determined based on the radi-

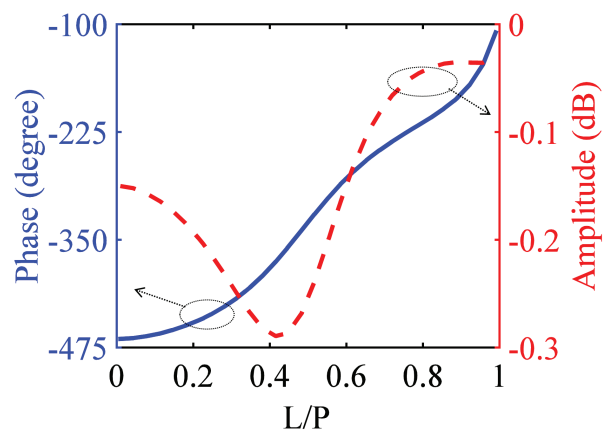

Figure 3: Reflection coefficient of the unit cell as a function of hole diameter at $10 \mathrm{GHz}$.

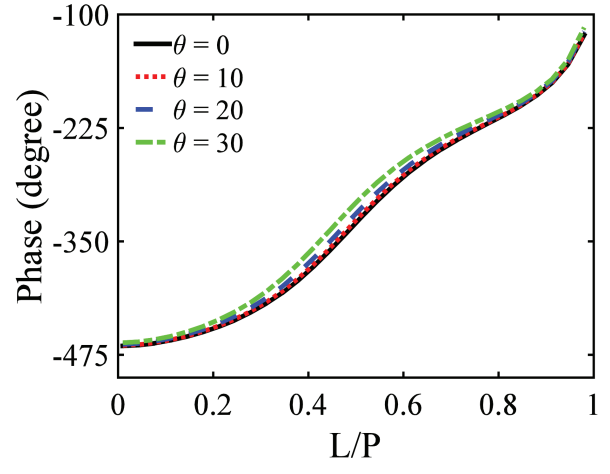

Figure 4: Reflection phase of the unit cell at $10 \mathrm{GHz}$ for different values of incidence angle.

ation pattern of the feed horn antenna and the reflectarray aperture efficiency considerations. The aperture efficiency of the reflectarray can be calculated as the product of the spill-over and illumination efficiencies, which are defined from the following expressions [21]:

$$
\begin{gathered}
\eta_{\text {spill-over }}=\frac{\iint_{\sigma} \vec{P} \cdot \overrightarrow{d s}}{\iint_{\Sigma} \vec{P} \cdot \overrightarrow{d s}} \\
\eta_{\text {illumination }}=\frac{1}{A} \frac{\left|\iint_{A} I(x, y) d x d y\right|^{2}}{\iint_{A}|I(x, y)|^{2} d x d y}
\end{gathered}
$$

In the above equations, $A$ is the aperture area of the reflectarray, $\sum$ is the entire spherical surface centered at the feed, $\sigma$ is a portion of the sphere, where the array aperture shares the same solid angle with respect to the feed, $\vec{P}$ is the Poynting vector of the feed defined by its power pattern as $\vec{P}=\frac{U(\theta, \varphi)}{r^{2}} \hat{a_{r}}$, and $I(x, y)$ is the amplitude distribution over the reflectarray aperture, which can be approximated by the feed power pattern and element pattern parameters as:

$$
I(x, y) \propto \frac{\sqrt{U(\theta, \varphi)}}{r} \cos ^{q_{e}}\left(\theta_{e}\right)
$$

In the case of our scenario, the feed radiates a null at the boresight direction, and its power pattern in the main lobe and nearest side lobes region can be approximated by $U(\theta, \varphi)=\sin 3 \theta, 0 \leq \theta \leq \pi / 3$.

To design the reflectarray, the aperture of reflectarray was divided into 14 concentric circular zones, and each zone was divided into 4 equal-angle sectors of same populating unit cells as shown in Fig. 1. The required phase shift for unit cells of each sector was then calculated as:

$$
\phi_{r_{m}}^{n}=k_{0}\left(F-\sqrt{F^{2}+r_{m}^{2}}\right)+\frac{2 \pi(n-1)}{N},
$$

where $r_{m}$ is the mean radius of zone $\mathrm{m}$, and $n$ is the sector number of that zone. 


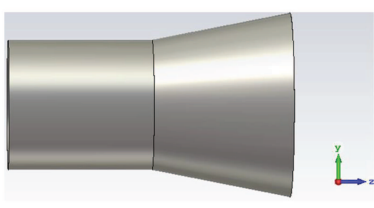

(a)

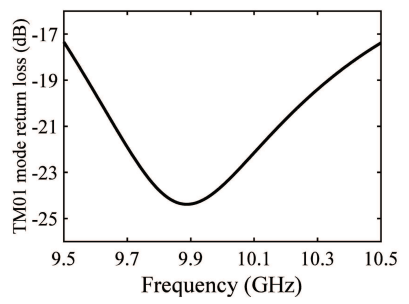

(c)

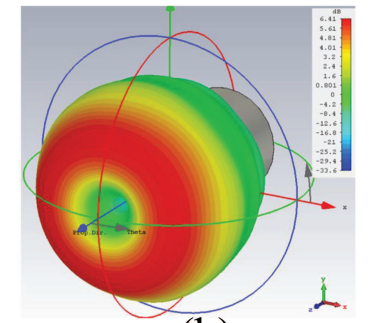

(b)

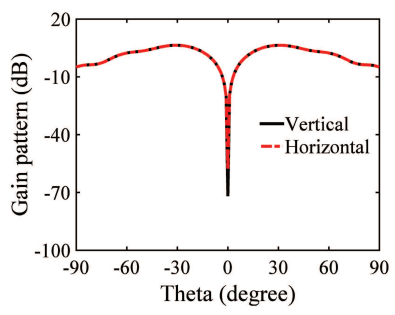

(d)
Figure 5: Characteristics of the designed conical horn antenna as a feed of the reflectarray aperture. (a) Schematic model. (b) Simulated three-dimensional doughnut-shaped pattern. (c) Simulated return loss for the $\mathrm{TM}_{01}$ mode. (d) Simulated radiation patterns for vertical and horizontal polarizations at $10 \mathrm{GHz}$.

The required phase distribution over the reflectarray aperture as well as the arrangement of unit cells populating different sectors, are shown in Fig. 6(a) and (b), respectively.

\section{Performance analysis and results}

Various techniques have been developed to calculate the radiation pattern of reflectarray antennas [22, 23, 24, 25, 26]. The phase only synthesis approach proposed in [23] was adopted here to formulate the problem due to its simplicity. The far-zone electric field radiated by the reflectarray aperture as a function of the direction cosines, $u=\sin \theta \cos \varphi$, and $v=\sin \theta \sin \varphi$ is expressed as follows:

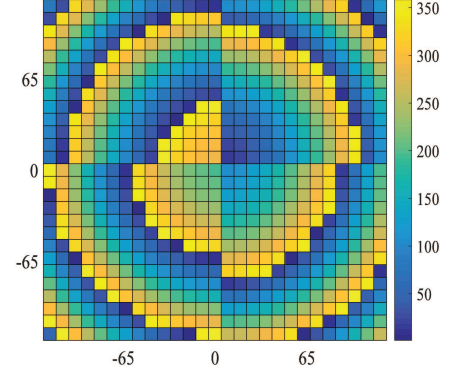

(a)

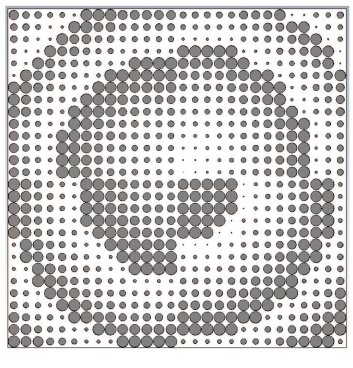

(b)
Figure 6: (a) Required phase shift distribution over the designed reflectarray aperture. (b) Corresponding dielectric unit cells configuration.

$$
\begin{aligned}
& \vec{E}(u, v)=\frac{j k_{0} e^{-j k_{0} r}}{2 \pi r}\left\{\hat{a}_{\theta}\left[\cos \varphi \tilde{E}_{x}(u, v)+\sin \varphi \tilde{E}_{y}(u, v)\right]\right. \\
& \left.+\hat{a}_{\varphi} \cos \theta\left[-\sin \varphi \tilde{E}_{x}(u, v)+\cos \varphi \tilde{E}_{y}(u, v)\right]\right\}
\end{aligned}
$$

where $(r, \theta, \varphi)$ are the standard coordinates of spherical system. The terms $\tilde{E}_{x}$ and $\tilde{E}_{y}$ are the spectral components of tangential radiated field over the reflectarray aperture, and are approximated by:

$$
\tilde{E}_{x / y}(u, v)=\zeta \sum_{b=-\frac{B}{2}}^{\frac{B}{2}-1} \sum_{a=-\frac{A}{2}}^{\frac{A}{2}-1} \Gamma_{x / y}^{a, b} \frac{E_{f e e d, x / y}^{a, b}}{\sqrt{F^{2}+x_{a}^{2}+y_{b}^{2}}} e^{j \Psi}
$$

where

$$
\begin{gathered}
\zeta=\frac{4 \pi^{2}}{k_{0}^{2} u v} \sin \left(\frac{k_{0} u d_{x}}{2}\right) \sin \left(\frac{k_{0} v d_{y}}{2}\right), \\
\Psi=-k_{0} \sqrt{F^{2}+x_{a}^{2}+y_{b}^{2}}+\phi_{R, x / y}^{a, b}+k_{0}\left(u a d_{x}+v b d_{y}\right)
\end{gathered}
$$

herein, $\left(x_{a}, y_{b}\right)$ are the coordinates of unit cell $(a, b), d_{x}$ and $d_{y}$ are the lateral dimensions of unit cell, $\mathrm{A}$ and $\mathrm{B}$ are the total number of unit cells along the $\mathrm{x}$ - and $\mathrm{y}$ - directions, $\Gamma_{x / y}^{a, b}$ and $\phi_{R, x / y}^{a, b}$ are the amplitude and phase of unit cell reflection coefficient, and $\frac{E_{f e e d, x / y}^{a, b}}{\sqrt{F^{2}+x_{a}^{2}+y_{b}^{2}}} e^{-j k_{0} \sqrt{F^{2}+x_{a}^{2}+y_{b}^{2}}}$ is the incident electric field at the unit cell, which is determined by the feed pattern at the location of unit cell $E_{\text {feed }, x / y}^{a, b}$, considering factors $\frac{1}{\sqrt{F^{2}+x_{a}^{2}+y_{b}^{2}}}$ for amplitude and $k_{0} \sqrt{F^{2}+x_{a}^{2}+y_{b}^{2}}$ for free space propagation phase shift to the unit cell position.

The full-wave simulation software CST Microwave Studio was used to examine the radiation performance of designed HPM reflectarray antenna. A comparison between the computed and simulated results of far-field radiation patterns for horizontally- and vertically polarized components, along the $\varphi=0$ cut at the design frequency of 10 $\mathrm{GHz}$ are presented in Fig. 7(a). The corresponding results along the $\varphi=90$ cut are given in Fig. 7(b). It can be seen that there is a good agreement between the theoretical and simulation results near the main lobe. As can be observed from these figures, the antenna radiates collimated beams of both horizontal and vertical polarizations at the direction of boresight in both principal planes. The amplitude and phase difference between these components over the frequency range of $8.75-10.75 \mathrm{GHz}$ were also simulated, and the results for $\varphi=0$ cut are shown in Fig. 8. As it can be seen, the amplitude and phase difference at $10 \mathrm{GHz}$ are about 0.2 $\mathrm{dB}$ and $81^{\circ}$, respectively, which means that the boresight null of azimuthally symmetric mode is transformed by the reflectarray aperture to a peak pattern with Left Handed Circular Polarization (LHCP) on the boresight direction. The simulated two-dimensional radiation patterns of the LHCP 

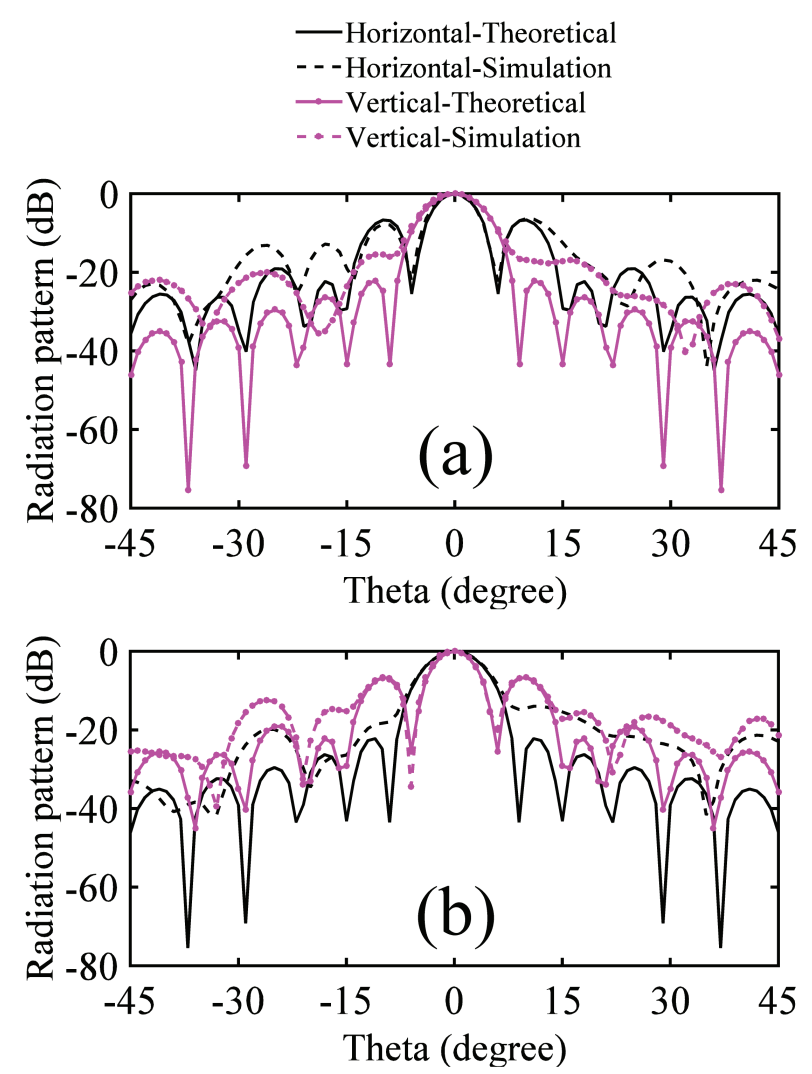

Figure 7: Radiation components of the designed reflectarray antenna at $10 \mathrm{GHz}$. (a) $\varphi=0$. (b) $\varphi=90$.

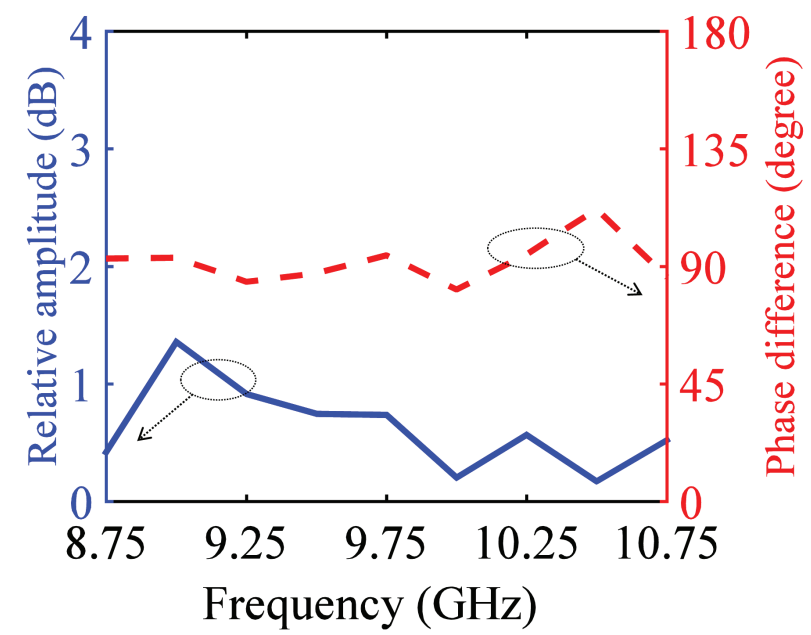

Figure 8: Amplitude and phase difference of horizontal polarization component relative to the vertical polarization as a function of frequency for $\varphi=0$ cut.

and RHCP (Right Handed Circular Polarization) components at the design frequency of $10 \mathrm{GHz}$ along the $\varphi=0$ cut are shown in Fig. 9. The peak gain is $20.2 \mathrm{~dB}$ and the antenna half power beam width and side lobe level (SLL) are about $6^{\circ}$ and $-12 \mathrm{~dB}$, respectively. The observed large amount of SLL is most likely due to the major discontinuity that exists at the reflectarray aperture in both horizontal and

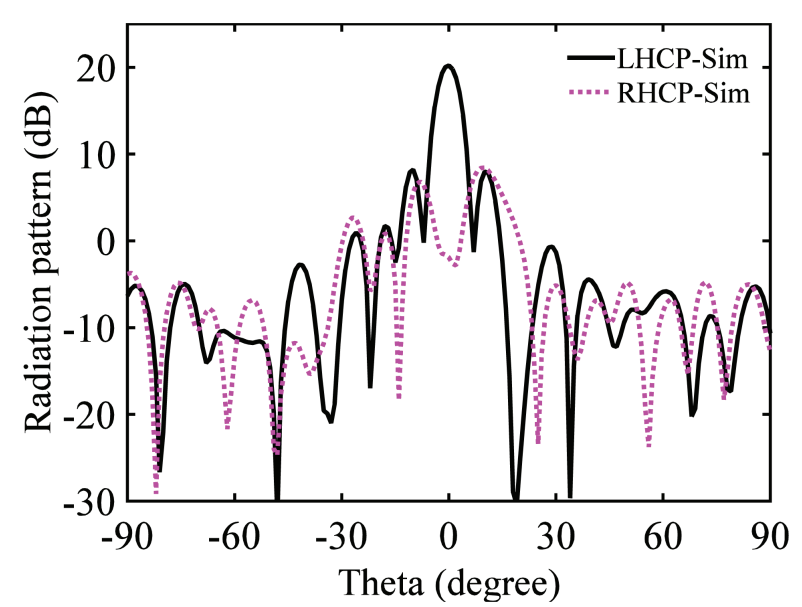

Figure 9: Simulated radiation patterns of the proposed HPM reflectarray antenna along the $\varphi=0$ cut.

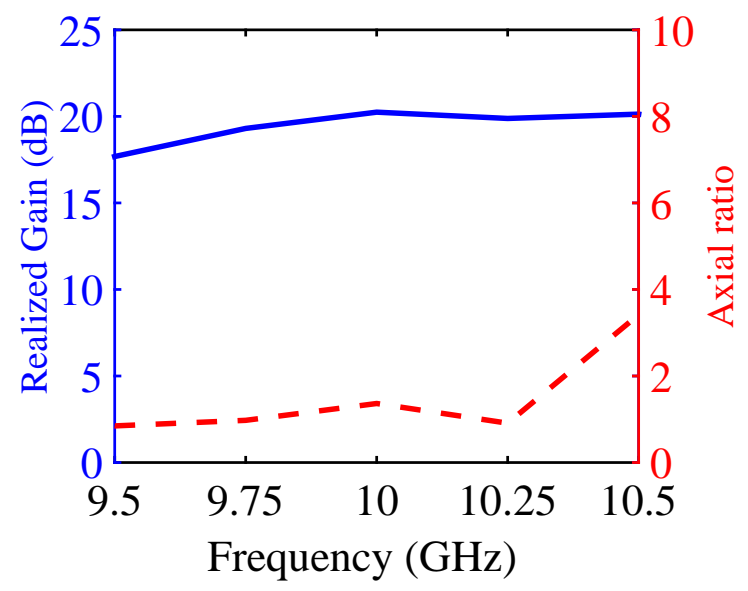

Figure 10: Simulated realized gain and axial ratio of the proposed HPM reflectarray antenna as a function of frequency.

vertical directions. The cross polarization level at the boresight direction is about $20 \mathrm{~dB}$ below that of the co-polarized radiation pattern. The simulated realized gain and axial ratio over the frequency range of $9.5-10.5 \mathrm{GHz}$ are shown in Fig. 10. The value of antenna axial ratio at $10 \mathrm{GHz}$ is 1.3 .

The power handling capacity of the proposed HPM reflectarray antenna can be investigated by using the simulated results of the electric field intensity inside its structure [8]. The distribution of electric field intensity inside the reflectarray plane for an input power of 1 watt is shown in Fig. 11. The maximum value of the electric field intensity is approximately $1811 \mathrm{~V} / \mathrm{m}$. Therefore, assuming the atmosphere breakdown threshold to be $3 \mathrm{MV} / \mathrm{m}$, the power handling capacity is equal to $\left(\frac{3 \times 10^{6}}{1811}\right)^{2} \approx 2.7 \mathrm{MW}$.

\section{Conclusions}

In this paper, the reflectarray concept was exploited to develop a high power antenna which accepts the azimuthally 

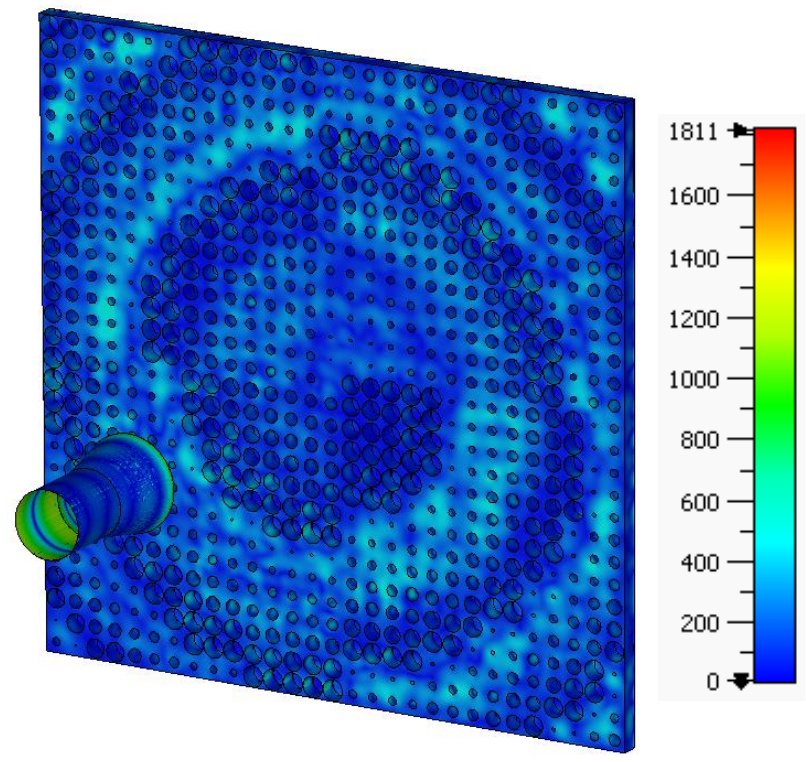

Figure 11: Electric field distribution of the proposed HPM reflectarray antenna.

symmetric output modes of high power microwave sources directly and radiates a collimated beam of circular polarization at the boresight direction. A $10 \mathrm{GHz}$ sample was designed and analyzed to demonstrate the feasibility of the proposed approach. The design exploited perforated dielectric substrate as the phase shifting unit cell to eliminate the problematic effects of metallic patterns at high levels of electromagnetic energy. The theoretical and simulation results are in good agreement, which shows the potential of proposed approach to be used in compact, high gain and high power antennas design for microwave regime.

\section{References}

[1] C.D. Taylor, D.V. Giri, High-Power Microwave Systems and Effects, Taylor \& Francis, New York, 1994.

[2] X.Q. Li, Q.X. Liu, A GW level high-power radial line helical array antenna, IEEE T. Antenn. Propag. 56: 2943-2948, 2008.

[3] J. Benford, J. Swegle, High-Power Microwaves, Artech House, Norwood, MA, 1992.

[4] B.M. Lee, W.S. Lee, Y.J. Yoon, J.H. So, X-band $\mathrm{TM}_{01}-\mathrm{TE}_{11}$ mode converter with short length for high power, Electron. Lett. 40: 1126-1127, 2004.

[5] X.Y. Wang, Y.W. Fan, T. Shu, C. Yuan, Q. Zhang, A high-efficiency tunable TEM-TE ${ }_{11}$ mode converter for high-power microwave applications, AIP $A d v$. 7: 035012-1-035012-6, 2017.

[6] S.N. Vlasov, I.M. Orlovax, Quasioptical transformer which transforms the waves in a waveguide having a circular cross section into a highly directional wave beam, Radiophys. Quant. El. 17: 115-119, 1974.
[7] C.C. Courtney, C.E. Baum, The coaxial beam-rotating antenna (COBRA): theory of operation and measured performance, IEEE T. Antenn. Propag. 48: 299-309, 2000.

[8] X.Q. Li, Q.X. Liu, 16-Element single-layer rectangular radial line helical array antenna for high-power applications, IEEE Antenn. Wirel. PR. 9: 708-710, 2010.

[9] S.B. Pottier, F. Hamm, D. Jousse, P. Sirot, F.T. Talom, R. Vézinet, High pulsed power compact antenna for high-power microwaves applications, IEEE T. Plasma Sci. 42: 1515-1521, 2014.

[10] S.R. Peng, C.W. Yuan, T. Shu, Z.Q. Li, Kind of highpower microwave radial line slot antenna, Electron. Lett. 49: 915-916, 2013.

[11] D. Berry, R. Malech, W. Kennedy, The reflectarray antenna, IEEE T. Antenn. Propag. 11: 645-651, 1963.

[12] J. Huang, J.A. Encinar, Reflectarray Antennas, John Wiley \& Sons, New Jersey, 2008.

[13] S.M.A.M.H. Abadi, K. Ghaemi, N. Behdad, Ultrawideband, true-time-delay reflectarray antennas using ground-plane-backed, miniaturized-element frequency selective surfaces, IEEE T. Antenn. Propag. 63: 534-542, 2015.

[14] D.M. Pozar, T.A. Metzler, Analysis of a reflectarray antenna using microstrip patches of variable size, Electron. Lett. 29: 657-658, 1993.

[15] L. Boccia, G. Amendola, G.D. Massa, Performance improvement for a varactor-loaded reflectarray element, IEEE T. Antenn. Propag. 58: 585-589, 2010.

[16] J. Huang, R.J. Pogorzelski, A Ka-band microstrip reflectarray with elements having variable rotation angles, IEEE T. Antenn. Propag. 46: 650-656, 1998.

[17] E. Carrasco, M. Barba, J.A. Encinar, Reflectarray element based on aperture-coupled patches with slots and lines of variable length, IEEE T. Antenn. Propag. 55: 820-825, 2007.

[18] J.H. Barton, All-dielectric frequency selective surface for high power microwaves, IEEE T. Antenn. Propag. 62: 3652-3656, 2014.

[19] M. Abd-Elhady, W. Hong, A Ka-band reflectarray implemented with a single-layer perforated dielectric substrate, IEEE Antenn. Wirel. PR. 11: 600-603, 2012.

[20] M.K.T. Al-Nuaimi, W. Hong, Discrete dielectric reflectarray and lens for E-band with different feed, IEEE Antenn. Wirel. PR. 13: 947-950, 2014. 
[21] A. Yu, F. Yang, A.Z. Elsherbeni, J. Huang, Y. RahmatSamii, Aperture efficiency analysis of reflectarray antennas, Microw. Opt. Technol. Lett. 52: 364-372, 2010.

[22] D.M. Pozar, D.M. Targonski, H.D. Syrigos, Design of millimeter wave microstrip reflectarrays, IEEE Antenn. Wirel. PR. 45: 287-296, 1997.

[23] J.A. Zornoza, J.A. Encinar, Efficient phase-only synthesis of contoured-beam patterns for very large reflectarrays, Int. RF Microw. C. E. 14: 415-423, 2004.

[24] M. Arrebola, Y. Alvarez, J.A. Encinar, Accurate analysis of printed reflectarrays considering the near field of the primary feed, IET Microw. Antenn. Propag. 3: 187-194, 2009.

[25] A. Capozzoli, C. Curcio, A. Liseno, G. Toso, Phaseonly synthesis of flat aperiodic reflectarrays, Prog. Electromagn. Res. 133: 53-89, 2013.

[26] M. Zhou, S.B. Sorensen, O.S. Kim, The generalized direct optimization technique for printed reflectarrays, IEEE T. Antenn. Propag. 62: 1690-1700, 2014. 